OPEN ACCESS

Edited by:

Yanmin Long,

Jianghan University, China

Reviewed by:

Junjie Zhu,

Nanjing University, China

Joaquim C. G. Esteves Da Silva,

University of Porto, Portugal

*Correspondence:

Mahdi Hesari

mhesari@uwo.ca

Zhifeng Ding

zfding@uwo.ca

Specialty section:

This article was submitted to

Nanoscience,

a section of the journal

Frontiers in Chemistry

Received: 04 July 2020 Accepted: 11 September 2020

Published: 10 November 2020

Citation:

Hesari M and Ding Z (2020) A

Perspective on Application of Carbon

Quantum Dots in Luminescence

Immunoassays.

Front. Chem. 8:580033.

doi: 10.3389/fchem.2020.580033

\section{A Perspective on Application of Carbon Quantum Dots in Luminescence Immunoassays}

\author{
Mahdi Hesari* and Zhifeng Ding * \\ Department of Chemistry, The University of Western Ontario, London, ON, Canada
}

Quantum dots (QDs) have been established in our daily life, for instance, in display screens and light-harvesting technologies, mainly owing to their peculiar opto-electronic features. However, toxicity of inorganic QDs, such as CdSe, CdTe, and perovskites, limits their applications in biological environments for medical diagnosis and bio-imaging purposes. A new generation of QDs called carbon quantum dots (CQDs) have been progressing rapidly over the past few years. CQDs have become as popular as other carbon-based nanomaterials such as carbon nanotubes (CNTs), due to their ease of preparation, ultra-small size, biocompatibility, and bright luminescence.

Keywords: carbon quantum dots, luminescence immunoassays, carbon-based nanomaterials, antibody and antigen detection, photoluminescence

\section{INTRODUCTION}

Carbon quantum dots (CQDs) are a newly developed division of carbon-based nanomaterials (e.g., nanodiamond, fullerenes, carbon nanotubes, graphene, and graphene oxide) which are environmentally friendly, chemically stable, and reasonably conductive (Lim et al., 2015; Cayuela et al., 2016; Liu et al., 2016; Namdari et al., 2017; Molaei, 2020). These basic properties have attracted a wide range of researchers to employ CQDs in various applications including biomedical and biotechnological applications (Namdari et al., 2017; Iravani and Varma, 2020), dye-synthesized solar cells (Gao et al., 2020), light-emitting devices, (Yuan et al., 2018; He et al., 2020) imaging and bioimaging (Zhu et al., 2012; Jiang et al., 2015a; Smith and Gambhir, 2017; Panwar et al., 2019; Liu Y. et al., 2020), electrochemical and electrochemiluminescence studies (Wang and Dai, 2015; Fiorani et al., 2019; Wang et al., 2019; Chen et al., 2020; Kour et al., 2020; Zhong et al., 2020), and sensing (Li et al., 2019; Huang et al., 2020; Molaei, 2020; Tajik et al., 2020). More importantly, CQDs possess outstanding optical features, including a bright and tunable luminescence emission within a wide range of wavelengths (Sun et al., 2006; Wang et al., 2017). The emission wavelength is easily altered by the size of CQDs and their composition, which can be obtained using different starting materials and/or synthesis methodology (Jiang et al., 2015a; Lim et al., 2015; Wang et al., 2017; Zhou et al., 2019; Huang et al., 2020; Liu Y. et al., 2020). Thus, this optical tunability of CQDs is being utilized for applications in photodetection platforms (Gao et al., 2016). In this perspective, we summarize the synthesis of CQDs and explain the influences of doping elements on the opto-electronics of these nanomaterials, while the main focus remains on their photochemistry. We further review the surface functional modification of CQDs, which enables researchers to exclusively couple them with a variety of antibodies, antigens, and proteins. The fundamental applications of CQDs in luminescent immunoassay detections of a broad range of biological samples are highlighted. In this perspective, we cover the most impactful applications of CQDs in immunoassays from 2014 to date. 


\section{SYNTHESIS OF CARBON QUANTUM DOTS}

The ease of preparation, characterization, and storage of CQDs have made these nanomaterials available and accessible for different proposes (Namdari et al., 2017; Iravani and Varma, 2020). Here, we summarize various synthesis methods, e.g., topdown and bottom-up methods, which are implemented to make CQDs, where the main difference between the two, are the availability and preference of the starting materials.

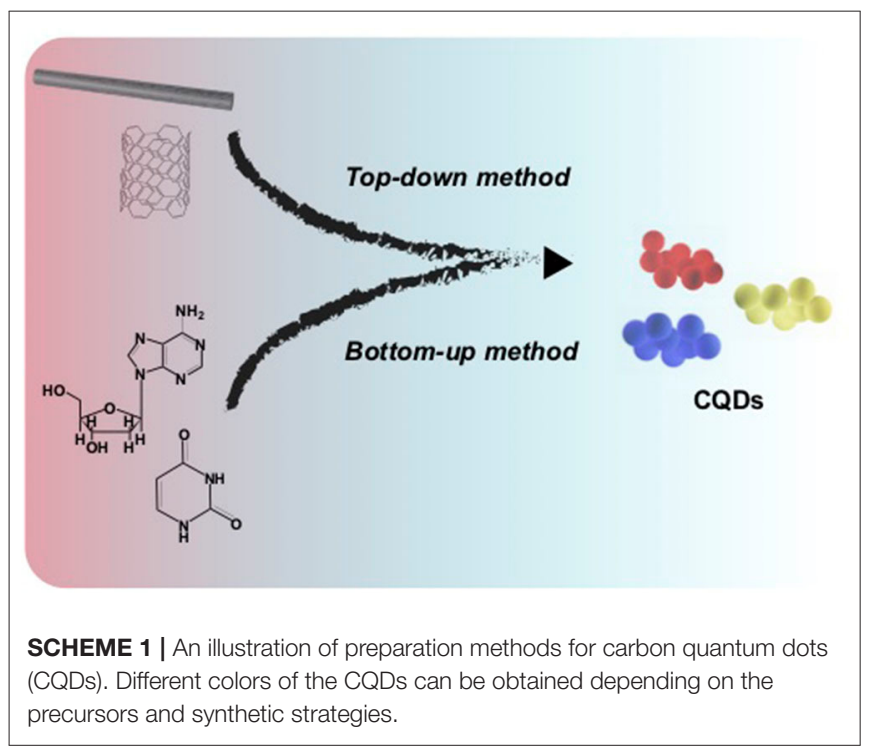

\section{Top-Down Method}

In this method a larger carbon source material has been used. For example, in an electrochemical oxidation (also known as exfoliation) approach a constant potential (e.g., $5 \mathrm{~V}$ ) is applied to a graphite rod immersed in an electrolytic solution $(\mathrm{Fu}$ et al., 2019). This technique, in fact, results in decomposition of a graphitic texture and the formation of smaller graphitic particles with highly oxidized surface functionalities. Our group has utilized multiwalled carbon nanotubes to generate graphene quantum dots via electro-exfoliation in a potentiodynamic process (Zhou et al., 2007). Alternatively, electrolysis of a carbon source, e.g., coke, using a two-electrode system including Pt and steel plates under constant current conditions, generates CQDs (He M. et al., 2018). Chemical oxidation of large organic structures e.g., polyamide systems and carbohydrates, in an acidic condition using a strong acid ultimately produces CQDs. For example chemical oxidation of tris(4-aminophenyl)amine (TAPA) in the presence of tert-butyl hydroperoxide (TBHP) and hydrochloric acid results in the formation of CQDs (Liu Y. et al., 2020). Furthermore, one can use high energy external resources such as ultrasonic (Dang et al., 2016), micro-plasma (Ma et al., 2019), or arc discharges (Arora and Sharma, 2014) to break down large organic molecules or other carbon sources into smaller pieces.

\section{Bottom-Up Method}

This method works by fusing and reconstructing small organic molecules to synthesize particles. There are different protocols
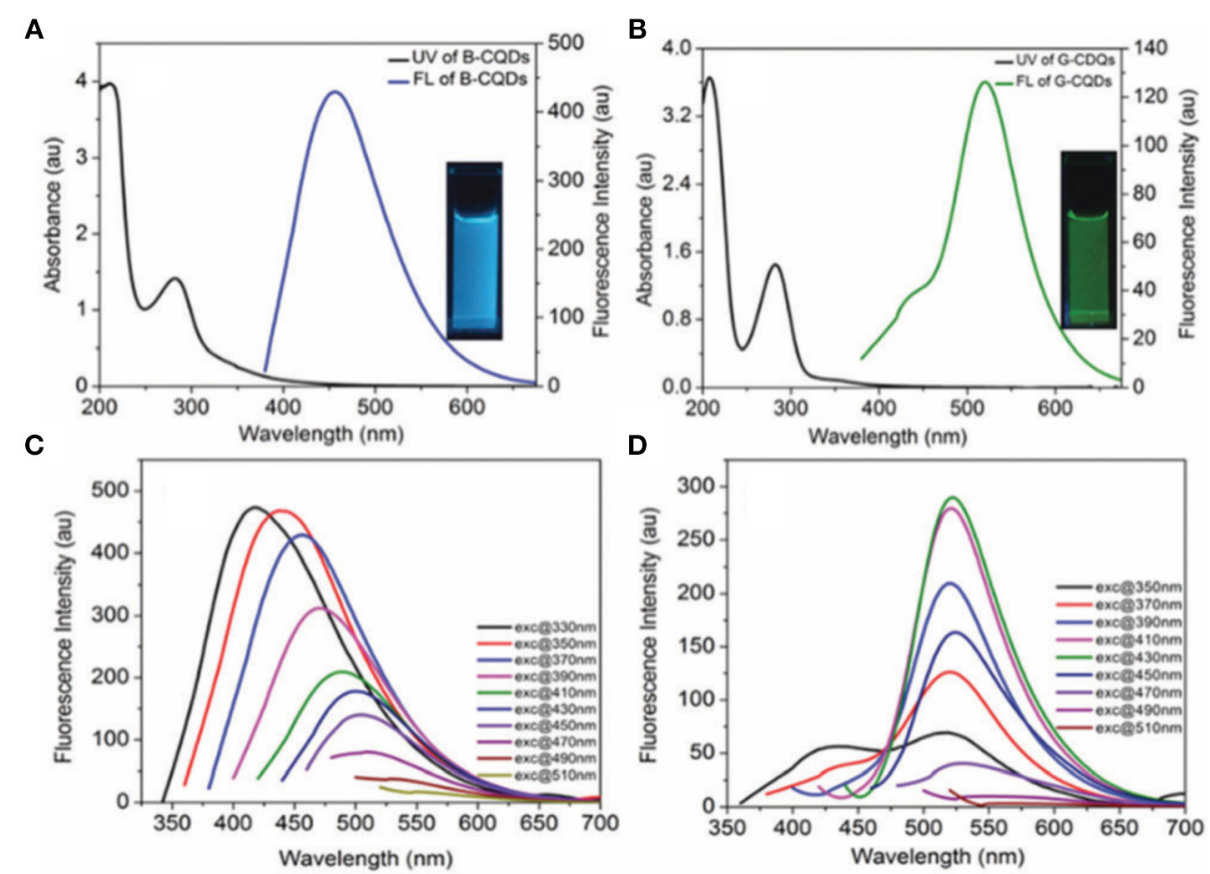

FIGURE 1 | Absorption and photoluminescence spectra of (A) blue and (B) green emissive CQDs. (C) and (D) represent the excitation-dependence spectroscopy of the blue and green CQDs at different excitation wavelengths. Panels reproduced from Singh et al. (2018). Copyright 2018, Royal Society of Chemistry. 
method) (e.g., microwave assisted, pyrolytic process, and template to synthesize CQDs following the bottom-up approach (Tajik et al., 2020); however the hydrothermal method is the most popular process owing to its easy setup and the broadness of useable reactive materials (Shen and Xia, 2014; Jiang et al., 2015b; Zhao et al., 2015; Wu et al., 2017; Mondal and Saha, 2019; Huang et al., 2020; Liu Y. et al., 2020). Indeed, in this approach small organic molecules, such as aspartic acid (Mondal and Saha, 2019), and cysteine (Huang et al., 2020), or easily decomposable organic biomasses such as garlic (Zhao et al., 2015), or grapefruit peel (Xiao et al., 2018) are used as starting materials. Practically, organic substances are dissolved in a solvent and transferred into a Teflon-lined autoclave and heated to a specific temperature to complete the reaction. Scheme 1 depicts a summary of CQD synthesis methods.

In all aforementioned methods, the final mixture, including residual starting materials and CQD particles with different sizes, are transferred into a dialysis bag or loaded onto a chromatographic column for further purification (Liu et al., 2018). Several techniques such as FT-IR, UV-visible, and photoluminescence (PL), X-ray photoelectron spectroscopy (XPS), and transmission electron microscopy (TEM) have been extensively employed to characterize CQDs (Liu et al., 2016; Fu et al., 2019; Ma et al., 2019; Liu Y. et al., 2020). For instance, FTIR and XPS have enabled researchers to identify and/or confirm surface functionality groups (e.g., $-\mathrm{OH},-\mathrm{NH}-,-\mathrm{SH}$ ), UV-visible spectroscopy along with PL have been used to investigate optical features of CQDs (vide infra). Furthermore, high-resolution TEM (HRTEM) helps to not only identify the size of individual particles, but it is also possible to recognize lattice fringes with an interplanar spacing.

\section{PHOTOPHYSICS OF CARBON QUANTUM DOTS}

Photoluminescence is a common and important property of CQDs, which empowers many researchers to make use of CQDs for various purposes such as light-emitting device fabrications (Yuan et al., 2018; He et al., 2020), and more widely for detecting diverse target species e.g., metal ion detection (Gao et al., 2016; Liu et al., 2017). The overall photophysical responses of CQDs in the course of light absorption and emission processes are based on an isolated network of $\pi$-bonding originating from $\mathrm{sp}^{2}$ carbon backbones. The critical point is that if the $\pi$-bonds form an extended network, similar to what is observed in carbon nanotubes (CNTs), graphite, and graphene, there is no or negligible light interaction. This may be due to the continuous non-radiative recombination among photogenerated electrons and holes (Lim et al., 2015). In fact, the light absorption in CQDs occurs almost in the wide range of the wavelength, however the dominant emission at a specific wavelength is governed by the population of a peculiar domain, and the inherent surface functionality. The excitation-dependent emission behavior is one of the typical photophysical characteristics of CQDs that originates from the surface state of these nanomaterials. For instance, Goel and co-workers prepared two fluorescent CQDs

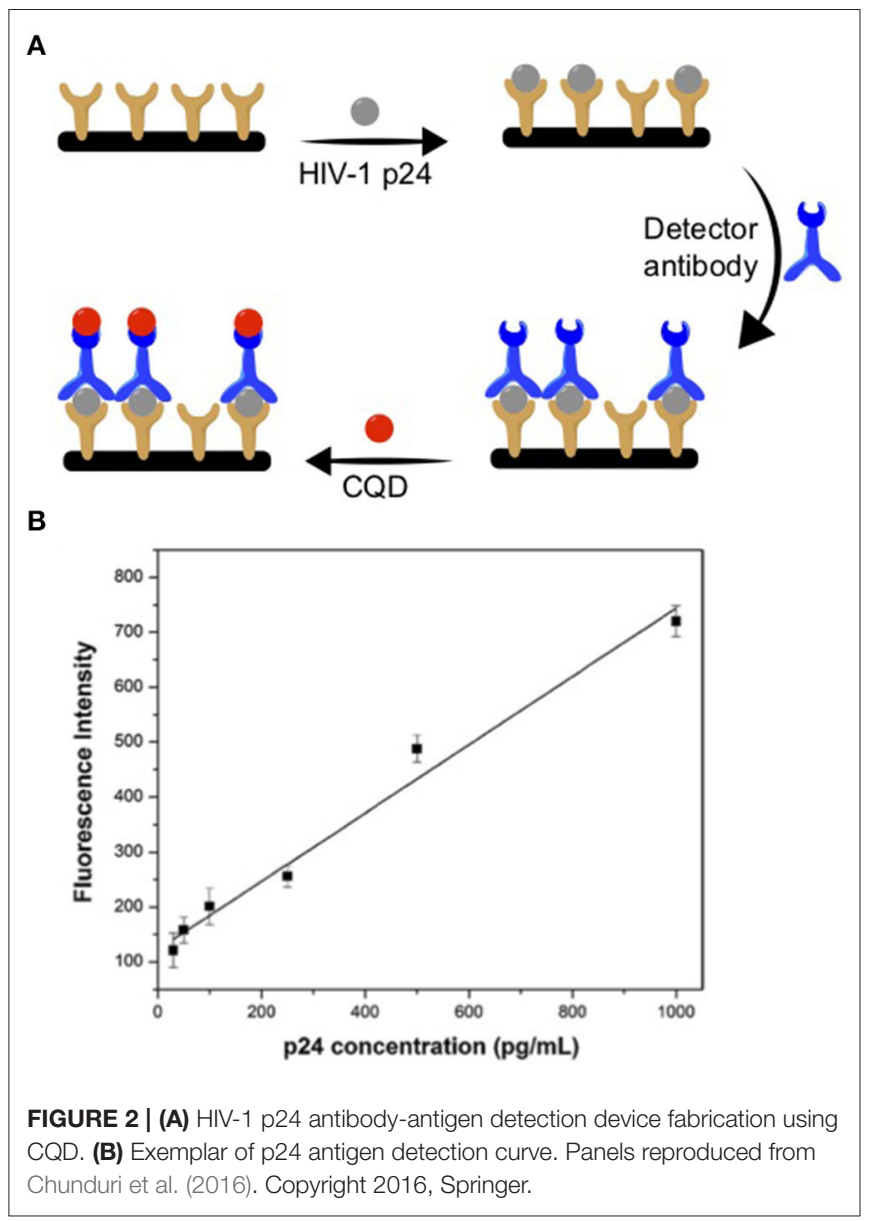

with blue and green emissions using hydrothermal and chemical oxidation methodologies, respectively (Singh et al., 2018). The UV-visible and PL spectra resulting from the blue emissive (B-CQD) and green emissive (G-CQD) materials are shown in Figures 1A,B with an absorption peak at $\sim 280 \mathrm{~nm}$ and PL emissions at 450 and $510 \mathrm{~nm}$, respectively. Interestingly, the BCQDs revealed an obvious excitation wavelength dependency at a 20-nm wavelength increment (Figure 1C), while G-CQDs show basically no dependency (Figure 1D). The excitation dependency property of CQDs is an extraordinary feature that leads to tunable photoluminescence nanomaterials. It is worth mentioning that the quantum efficiency and tunablity of PL are improved by using a passivation agent such as poly(propionyl ethyleneimineco-ethyleneimine) (PPEI-EI) (Sun et al., 2006), or polyethylene (PE) (Fernandes et al., 2020). In fact, the polymeric layer(s) could extend emission life time, and enhance the PL quantum yield, that depends on $\pi-\pi^{*}$ and $n-\pi^{*}$ transitions across the carbon backbone and also surface functionalities (Dimos, 2018).

\section{Effect of Doping and Surface Alteration on Photophysical Features}

The replacement of the main carbon backbone by a soft element e.g., S (Yang et al., 2016; Yao et al., 2019), Se (Yang et al., 2014), N (Jiang et al., 2015b; Chen et al., 2016; Carolan et al., 
A

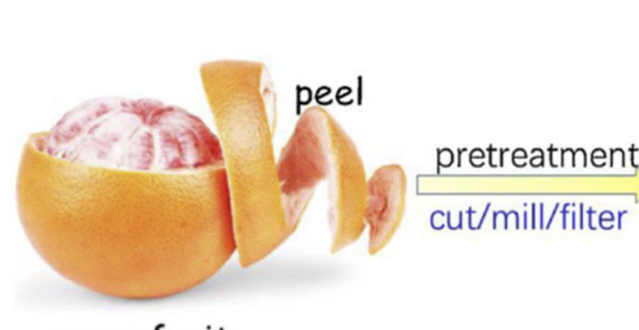

grapefruit

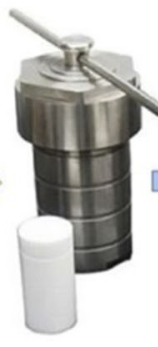

hydrothermal carbonization

$190^{\circ} \mathrm{C}, 12 \mathrm{~h}$

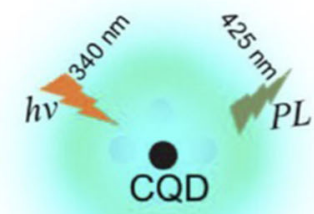

carbon quantum dot

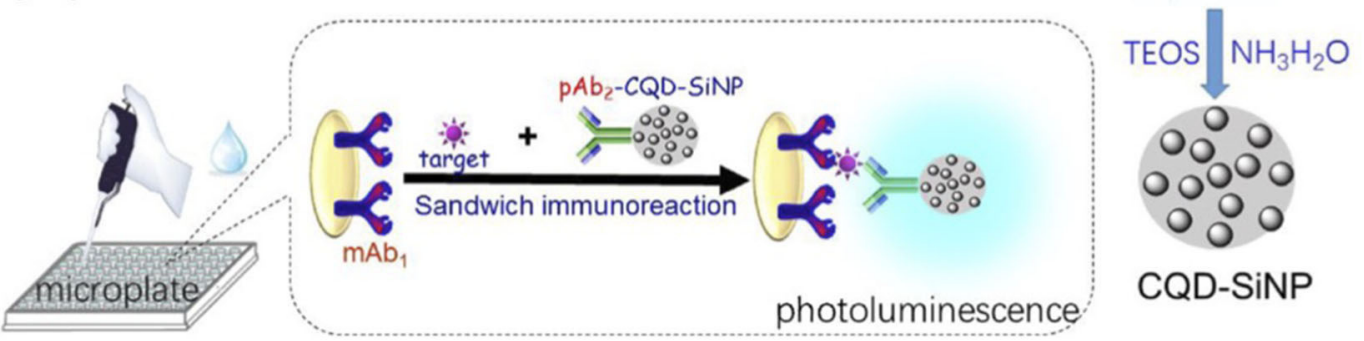

B

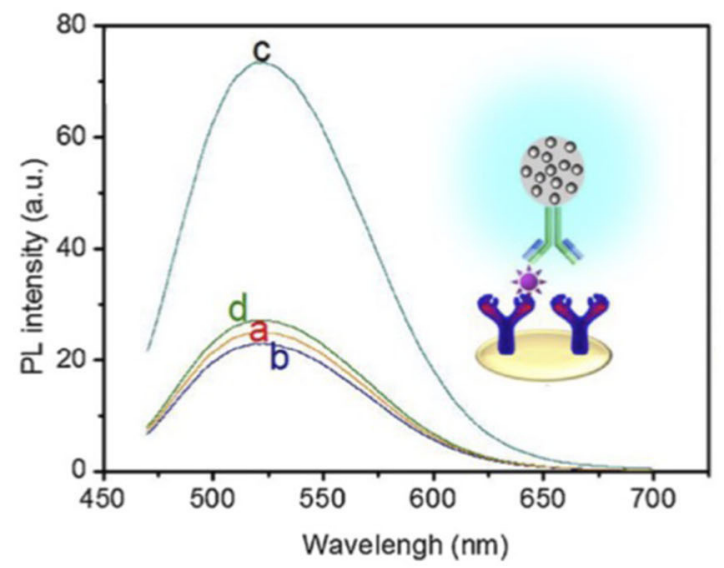

C

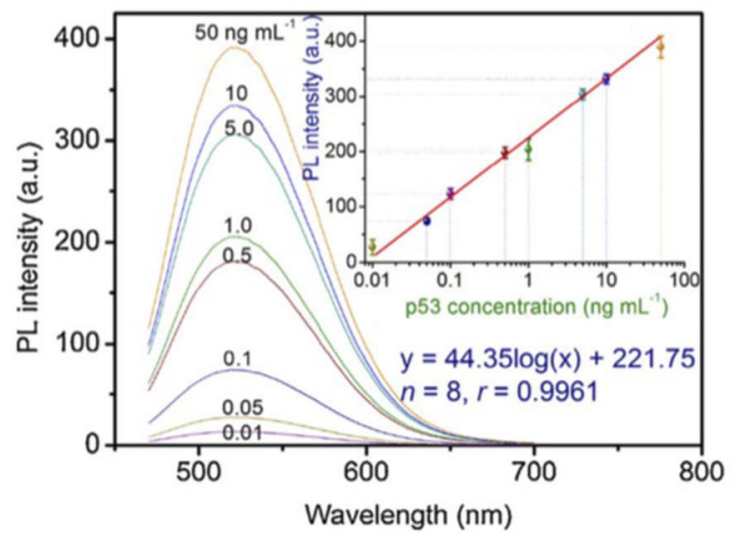

FIGURE 3 | (A) Preparation, encapsulation, and assembly steps of CQDs used for p53 protein luminescence detection. (B) PL emission spectra of (a) mAb

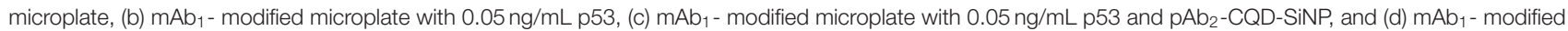
microplate with $\mathrm{pAb}_{2}-\mathrm{CQD}$-SiNP. (C) Luminescence emission spectra of the designed immunoassay for the various p53 standards along with the corresponding calibration curve. Panels reproduced from Xiao et al. (2018). Copyright 2018, Elsevier.

2017; He S. et al., 2018; Yao et al., 2019), P (Venkateswara Raju et al., 2020), B (Wei et al., 2020), or both $\mathrm{N}$ and S (Zhang et al., 2018), and a hard element e.g., Fe (Zhuo S. et al., 2019) and Ag (Zhuo S. J. et al., 2019) results in the formation of a doped CQD. For example, Raju et al., autoclaved tri-sodium citrate in the presence of phosphoric acid at $180^{\circ} \mathrm{C}$ for $12 \mathrm{~h}$ resulting in the formation of a $\mathrm{p}-$ doped CQD, which was further used to detect $\mathrm{Cu}^{2+}$ ions (Venkateswara Raju et al., 2020). A yellow emission B and $\mathrm{N}$ CQD was also synthesized by mixing o-phenylenediamine with boric acid in an autoclave apparatus. The resulting co-doped CQD revealed a $\sim 14 \%$ PL quantum yield (Wei et al., 2020). Liu et al. produced a triply-doped CQD consisting of $\mathrm{B}, \mathrm{N}$, and $\mathrm{S}$ in a one-pot hydrothermal reaction with an emission at $\sim 600 \mathrm{~nm}$ (Liu et al., 2017). It has also been shown that the more electronegative the doping element, the more red-shift occurs in the luminescence emission (Yang et al., 2014). Overall, doping approaches contribute significantly to the improvement of the PL quantum efficiency and provide alternations of the emission wavelengths, by creating the population of an electron rich domain and sometimes more defects. Xu et al. explored the effect of edge functionality on the luminescent emissions of CQDs. They altered the inherent functional groups such as $\mathrm{COOH}$ and $-\mathrm{C}=\mathrm{O}$ to phosphate, sulfite, and amine by treating CQDs in the presence of phosphoric acid, sulfuric acid, and nitric acid, respectively. They observed that when the $-\mathrm{COOH}$ and $\mathrm{C}=\mathrm{O}$ groups were presence at the edge of CQDs, $\mathrm{PL}$ quantum efficiency reached to $\sim 9 \%$, while introducing the $-\mathrm{PO}_{4}^{2-},-\mathrm{SO}_{3}^{-}$, and $-\mathrm{NH}_{2}$ groups improved the PL quantum efficiency to 10.3 , 29.7 , and $18.7 \%$, respectively. 

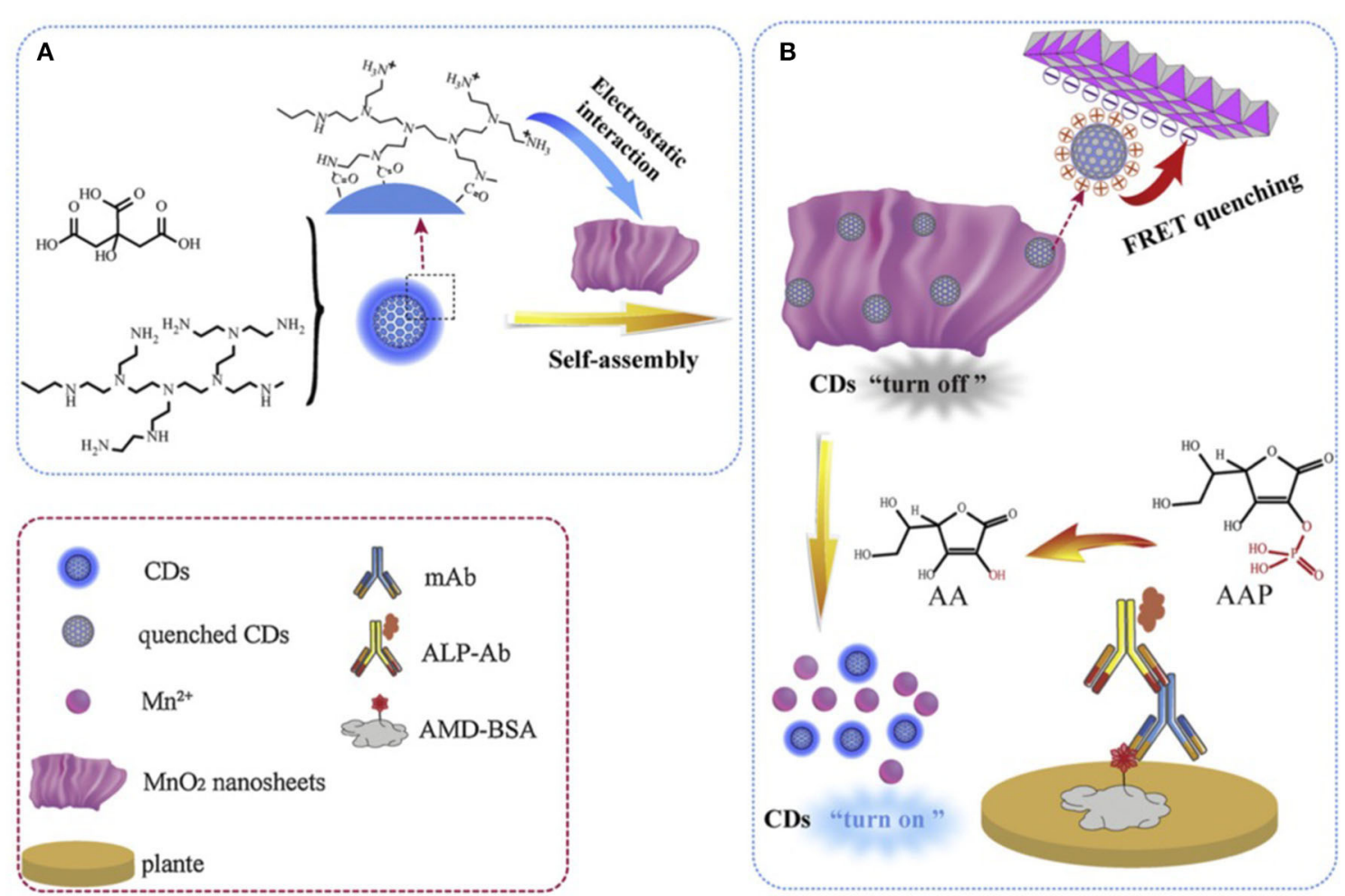

FIGURE 4 | (A) Synthesis and (B) fabrication of designed luminescence immunoassay to detect amantadine (AMD). Panels reproduced from Dong et al. (2019b). Copyright 2019, Elsevier.

\section{APPLICATION OF CARBON QUANTUM DOTS IN IMMUNOASSAYS}

Ease of preparation, chemical and biological compatibility, welldefined luminescence signal with tunable wavelength options, diverse surface functionality for further modification, low electrical resistance, and acceptable conductivity, all make CQDs an exceptional candidate for detection technology. There are several designed detecting systems that are constructed around CQD luminescence signals measuring a diverse range of analytes in biological environments, for instance, aptasensor for ochratoxin A detection by Bi et al. (2020). In this section, we focus on recent developments on using CQDs for immunoassays. In fact, CQDs have been mainly utilized in detection platforms owing to their stable luminescence emission (Xiao et al., 2018; Dong et al., 2019a; Yao et al., 2019; Alarfaj et al., 2020; Liu G. et al., 2020). In the past decade, luminescence-based immunoassays have become powerful tools in the field of biological and clinical diagnoses, and microbial investigations. These nanomaterials have been fabricated in point-of-care (POC) systems along with antigen-antibody binding interactions and enzyme-substrate reactions. Their optical properties have been altered and applied through different approaches in which the optical signal was quenched, recovered, or enhanced for the purpose of detection.
For example, Chunduri et al. designed an early stage detection of HIV through a POC system (Chunduri et al., 2016). They synthesized nitrogen doped (N-doped) CQDs using citric acid and ethylenediamine as carbon and nitrogen precursors via a one-pot hydrothermal reaction. These CQDs were decorated with streptavidin (CQD-SA) and have been used as fluorescent labels to measure the HIV-1 p24 antigen on both microwell plates and microfluidic chips. The microfluidic devices were machined on a $100 \times 100 \times 1.5 \mathrm{~mm}$ acrylic plaque that was used as a template for polydimethylsiloxane (PDMS) to replicate the microchip pattern for further use. The next step for the device was loading the captured antibody into micro channels with a micropipette and incubating it. Next, the HIV-1 p24 antigen sample solutions were loaded. The loosely bound bonded HIV1 p24 was then washed away with a buffer. Later, the detector antibody was added to each channel to assemble the antibodyantigen-antibody sandwich immunocomplex. Finally, the CQDSA solution was loaded into each channel and the streptavidinbiotin coupling reaction was allowed to complete. Figure 2A shows a summary of the fabrication process. The fluorescent signals from the sandwich immunocomplex were then recorded as an analytical signal using a spectraMax M5 fluorescence reader. Figure 2B shows an exemplary p24 measurement using the device based on a CQD luminescence signal in a $\mathrm{pg} / \mathrm{mL}$ detection range with an $R^{2}=0.9737$ linear correlation. The 
inexpensive preparation of CQD and the stable luminescence signal made this device cheaper and more efficient than conventional ELISA immunoassays. The advantage of this type of fabrication is its ease of assembly with minimal required steps.

Xiao et al. prepared water-soluble CQDs using grapefruit peel treated under hydrothermal conditions at $190^{\circ} \mathrm{C}$ for $12 \mathrm{~h}$ (Xiao et al., 2018). The resulting CQDs with an average size of $4.2 \pm 0.1 \mathrm{~nm}$ emit light at $425 \mathrm{~nm}$ after excitation at $340 \mathrm{~nm}$. Figure 3A represents the summary of the preparation steps. In order to boost the luminescence signal, the CQDs were mixed with a silica precursor. The resulting CQDs-silica nanoparticle (CQD-SiNP), with an average size of $\sim 16 \mathrm{~nm}$, was functionalized with the polyclonal anti-p53 antibody (CQD-SiNP-pAb 2 ). The luminescence wavelength of the CQD-SiNP was red shifted owing to the inner-filter effect. To fabricate the detection system, a microplate was decorated with the monoclonal mouse antip53 antibody $\left(\mathrm{mAb}_{1}\right)$. To join CQD-SiNP-pAb2 and the mAb1 the target protein, p53 was added into the microplate. They found that a strong signal could be produced by adding 0.05 ng $\mathrm{mL}^{-1}$ p53 and $\mathrm{pAb}_{2}$-CQD-SiNP into a $\mathrm{mAb}_{1}$ - modified microplate (Figure 3B). There is a linear relationship (0.01$50 \mathrm{ng} / \mathrm{mL}$ ) between the detected signal and the added known concentration of the target protein, p53 (Figure 3C). They further found that this method with a limit of detection (LOD) of $2.7 \mathrm{pg} / \mathrm{mL}$ works as good as a commercial ELISA kit with a LOD of $65 \mathrm{pg} / \mathrm{mL}$, while it is less expensive to fabricate. Indeed, the luminescence signal improvement facilitated the detection sensitivity and responsivity.

In another strategy, luminescence emission quenching of CQDs driven by antibody interaction was also employed in immunoassay measurements. Yao et al. synthesized S- and $\mathrm{N}$-doped CQDs (S/N-CQDs) with luminescence emissions at $405 \mathrm{~nm}$ upon excitation at $350 \mathrm{~nm}$ (Yao et al., 2019). The luminescence enhancement of the S/N-doped CQDs was investigated. They proposed a potential mechanism for luminescence improvement for S/N-doped CQDs that was based on a higher population of excited electrons of the doped CQD relative to non-doped ones along with a red-shift emission. They found that if the S/N-doped CQDs coupled with an antibody, e.g., clenbuterol (Clen) antibody $(\mathrm{Ab})$ through amine-amine coupling, the original luminescence intensity of the S/N-doped CQDs would be quenched. Thus, one can use this luminescence signal change as an analytical detection signal to measure Clen in biological environments. In fact, attaching and de-attaching the CQD to the antibody created the luminescence signal difference, $\Delta \mathrm{F}$. To establish the signal alternation in the presence and absence of Clen, various concentrations of Clen were added to a solution of S-N-CQD solution and the corresponding luminescence spectra were recorded. The extracted $\Delta \mathrm{F}$ showed a linear relationship vs. the concentration of Clen, that is an indication of the strong potential application of the strategy to detect Clen in various samples.

The sensitivity and selectivity of immunoassay measurements based on CQD luminescence signals, and the importance of residual harmful chemicals detection in food chemistry have encouraged Shen and co-workers to expand CQD application in the food industry (Dong et al., 2019b). In their study, the
A
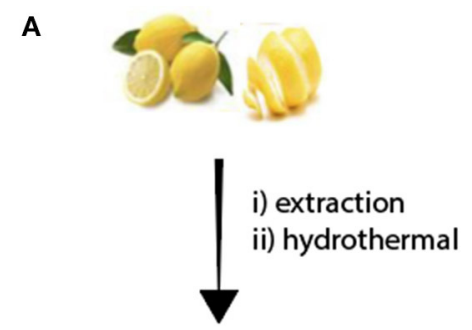

B
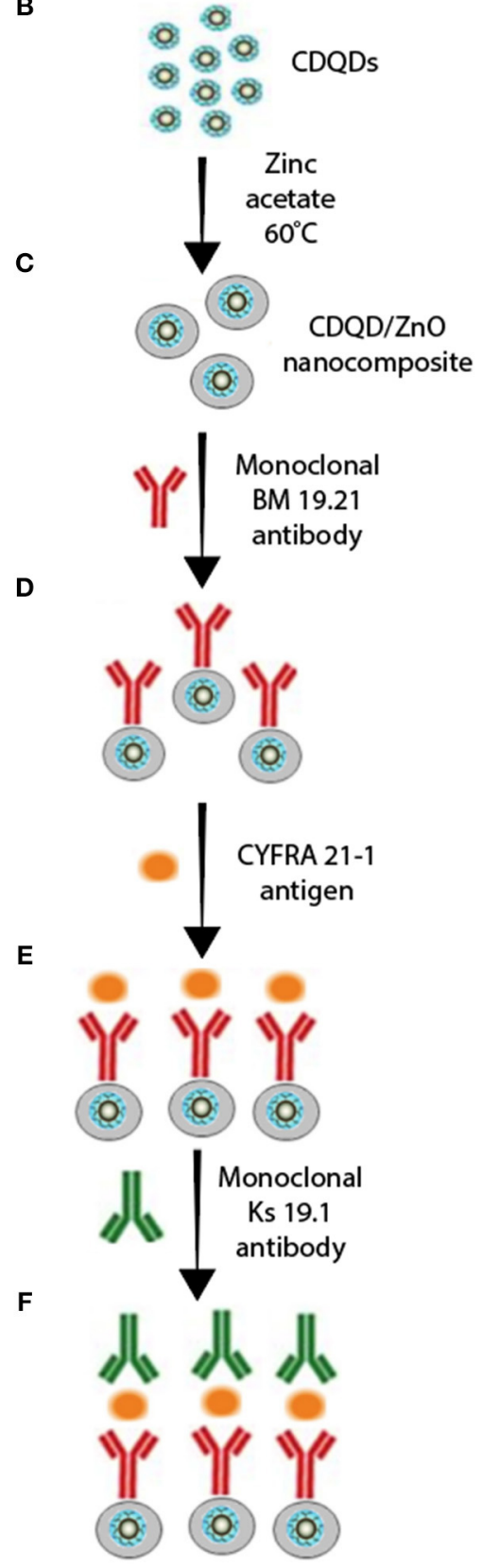

SCHEME 2 | Luminescence immunoassay based on CQDs/ZnO nanocomposite and antibody-antigen-antibody system. (A,B) CDQD synthesis, (C) ZnO coated CDQD, (D) decorated CDQD/ZnO with 8M 19.21 antibody, (E) fabricated CDQD/ZnO/antibody with CYFRA 21.1 antigen, (F) final assembly of $\mathrm{CDQD} / \mathrm{ZnO} /$ antibody/antigen/antibody assay. Panel reproduced from Alarfaj et al. (2020). Copyright 2020, Springer. 
luminescence signal recovery was employed as a detection signal for amantadine (AMD). The surface functionalized CQD was prepared by mixing citric acid and polyethyleneimine branched $(\mathrm{PEI}, \mathrm{MW}=10,000)$ in a Teflon-lined autoclave, which was heated at $180^{\circ} \mathrm{C}$ for $6 \mathrm{~h}$. The resulting CQDs with charged polymeric amine-based coating ( $\mathrm{p}$-CQDs) emits at $\sim 450 \mathrm{~nm}$ with a quantum yield of $41.7 \%$ and an average size of $8 \mathrm{~nm}$. Owing to the presence of ammonium moieties on the surface of CQDs they interacted strongly with the negatively charged $\mathrm{MnO}_{2}$ nanosheets layer (Figure 4A). The resulting nanocomposite (i.e., $\mathrm{p}-\mathrm{CDs} @ \mathrm{MnO}_{2}$ ) quenched the original luminescence of CQDs via Forster resonance energy transfer (FRET) between CQDs and $\mathrm{MnO}_{2} \mathrm{NSs}$. A further luminescence-based immunoassay was fabricated by placing an antigen, AMD-BSA (Wang et al., 2018), on a microplate. In fact the AMD-BSA acted as an anchor to build the rest of the assay (Figure 4B).

Next an antibody, anti-AMD mAbs, was added to react with the absorbed BSA-AMD antigen. Then an ALP-labeled secondary antibody was introduced. This strategy could result in a lower amount of the required ALP-labeled secondary antibody as a reactive catalysis site. In fact, the ALP-labeled secondary antibody acted as a catalyst for the hydrolysis of 2-phosphoascorbic acid trisodium salt (AAP) to ascorbic acid (AA). At this stage AA triggered p-CDs@ $\mathrm{MnO}_{2}$ dissociation, that recovered the luminescence of the CQDs which served as the detection signal. Indeed, this is a sophisticated approach for the detection and measurement of an important food poisoning chemical. They also found that the designed system was more sensitive to AA rather than AAS. Further they assessed the selectivity of the immunoassay in the presence of a series of non-targeted chemicals such as ribavirin, moroxydine, acyclovir, acetaminophen, chloramphenicol, enrofloxacin, and sulfamethazine. This system responded to $0.048-1.1 \mathrm{ng} \mathrm{mL}^{-1}$ of AND with a LOD of $0.035 \mathrm{ng} \mathrm{mL}^{-1}$.

Recently, Oraby and co-workers reported a nanocomposite consisting of CQD (size $\sim 5 \mathrm{~nm}, \lambda_{\text {abs. }}=224 \mathrm{~nm}$, and $280 \mathrm{~nm}, \lambda_{\text {em }}=452 \mathrm{~nm}$ ) decorated with zinc oxide (i.e., $\mathrm{CQD} / \mathrm{ZnO}, \lambda_{\text {abs. }}=370 \mathrm{~nm}, \lambda_{\text {em }}=520 \mathrm{~nm}$ ) (Alarfaj et al., 2020) (Schemes 2A,B). The resulting highly stable CQD/ZnO composite (Scheme 2) was further decorated with a nonconjugated monoclonal BM 19.21 antibody via the reaction of the surface carboxylic groups with amine sites of the peptide amide of the antibody backbone in the presence of N-hydroxysuccinimide (NHS) and carbodiimide hydrochloride (EDC) (Scheme 2D). The CQDs/ZnO-BM 19.21 luminescence immunoassay was further constructed and utilized to detect lung cancer at early stages using the antigen cytokeratin-19 fragment (CYFRA 211) as a tumor marker (Scheme 2E). The antibody-antigenantibody immunoassay sandwich was completed by introducing the monoclonal Ks 19.1 antibody (Scheme 2F).

It was found that CQDs/ZnO-BM 19.21 immunoassay systems work within a wide range of linear detection (i.e., 0.01-100 ng. $\mathrm{mL}^{-1}$ ) with a LOD of $0.008 \mathrm{ng} \mathrm{mL} \mathrm{mh}^{-1}$ wh is easier to use than other techniques such as enzyme and electrochemiluminescence (ECL) immunoassays. For instance, in order to utilize an electrochemical or ECL immunoassay sensor, the modified CQDs need to be assembled on the surface of an electrode. This, occasionally, results in reduced stability in the immunosensor. Further specific tailoring of the CQDs functionalities is needed (Chen et al., 2020; Zhong et al., 2020).

\section{PERSPECTIVE AND FUTURE WORK}

Immunoassay detection is a well-established technique to measure various target molecules in many biological matrices. There are a vast number of approaches to design a selective and sensitive immunoassay system, while the unique inherent prosperities of CQDs have assisted researchers to achieve discriminatory systems with much higher precision when detecting crucial analytes. For example, as we summarized in the current article, CQD luminescence emission provides a well-defined detecting signal that is sensitive to reaction and interaction in the analyte solution. It is important to highlight that the variety of original chemical functionality such as $-\mathrm{OH},-$ $\mathrm{COOH},-\mathrm{C}=\mathrm{O}$, and upon doping, $-\mathrm{NH}_{2},-\mathrm{SO}_{3}^{-},-\mathrm{PO}_{4}^{2-}$ provide straightforward and easy access platforms that outperform many other options. For instance, upon the appearance of COVID19 as a worldwide pandemic, one can use CQDs along with an antibody-antigen-antibody strategy to detect COVID-19 in various media (Nabel and Shiver, 2019; Pinto et al., 2020; Shi et al., 2020). Here we highlighted the most recent advanced procedures on the luminescence features of CQDs. Alternatively, based on easy modifications with antigens and antibodies and good conductivity of CQDs one can develop electrochemical and electrochemiluminescence (ECL) studies toward various detecting proposes including immunoassay sensing (Zhou et al., 2014; Chen et al., 2020). This approach can be further expanded in solid-state electrochemical and ECL investigations by employing surface functionalities of CQDs and generate background free signals toward detection.

\section{DATA AVAILABILITY STATEMENT}

The original contributions presented in the study are included in the article/supplementary material, further inquiries can be directed to the corresponding author/s.

\section{AUTHOR CONTRIBUTIONS}

$\mathrm{MH}$ and $\mathrm{ZD}$ defined the topic and wrote the manuscript.

\section{FUNDING}

This work was supported by the Natural Sciences and Engineering Research Council (NSERC) of Canada (DG RGPIN-2018-06556 and SPG STPGP-2016-493924), the Canada Foundation of Innovation/Ontario Innovation Trust (CFI/OIT, 9040), the Premier's Research Excellence Award (PREA, 2003), and The University of Western Ontario. 


\section{REFERENCES}

Alarfaj, N. A., El-Tohamy, M. F., and Oraby, H. F. (2020). New immunosensingfluorescence detection of tumor marker cytokeratin-19 fragment (CYFRA 211) via carbon quantum dots/zinc oxide nanocomposite. Nanoscale Res. Lett. 15:12. doi: 10.1186/s11671-020-3247-9

Arora, N., and Sharma, N. N. (2014). Arc discharge synthesis of carbon nanotubes: comprehensive review. Diam. Relat. Mater. 50, 135-150. doi: 10.1016/j.diamond.2014.10.001

Bi, X., Luo, L., Li, L., Liu, X., Chen, B., and You, T. (2020). A FRET-based aptasensor for ochratoxin A detection using graphitic carbon nitride quantum dots and $\mathrm{CoOOH}$ nanosheets as donor-acceptor pair. Talanta 218:121159. doi: 10.1016/j.talanta.2020.121159

Carolan, D., Rocks, C., Padmanaban, D. B., Maguire, P., Svrcek, V., and Mariotti, D. (2017). Environmentally friendly nitrogen-doped carbon quantum dots for next generation solar cells. Sustain. Energy Fuels 1, 1611-1619. doi: 10.1039/C7SE00158D

Cayuela, A., Soriano, M. L., Carrillo-Carrión, C., and Valcárcel, M. (2016). Semiconductor and carbon-based fluorescent nanodots: the need for consistency. Chem. Commun. 52, 1311-1326. doi: 10.1039/C5CC07754K

Chen, S., Chen, X., Xia, T., and Ma, Q. (2016). A novel electrochemiluminescence sensor for the detection of nitroaniline based on the nitrogen-doped graphene quantum dots. Biosens. Bioelectron. 85, 903-908. doi: 10.1016/ j.bios.2016.06.010

Chen, Y., Cao, Y., Ma, C., and Zhu, J.-J. (2020). Carbon-based dots for electrochemiluminescence sensing. Mater. Chem. Front. 4, 369-385. doi: 10.1039/C9QM00572B

Chunduri, L. A. A., Haleyurgirisetty, M. K., Patnaik, S., Bulagonda, P. E., Kurdekar, A., Liu, J., et al. (2016). Development of carbon dot based microplate and microfluidic chip immunoassay for rapid and sensitive detection of HIV-1 p24 antigen. Microfluid Nanofluid 20:167. doi: 10.1007/s10404-016-1825-Z

Dang, H., Huang, L.-K., Zhang, Y., Wang, C.-F., and Chen, S. (2016). Large-scale ultrasonic fabrication of white fluorescent carbon dots. Ind. Eng. Chem. 55, 5335-5341. doi: 10.1021/acs.iecr.6b00894

Dimos, K. (2018). Tuning carbon dots' optoelectronic properties with polymers. Polymers 10:1312. doi: 10.3390/polym10121312

Dong, B., Li, H., Mujtaba Mari, G., Yu, X., Yu, W., Wen, K., et al. (2019a). Fluorescence immunoassay based on the inner-filter effect of carbon dots for highly sensitive amantadine detection in foodstuffs. Food Chem. 294, 347-354. doi: 10.1016/j.foodchem.2019.05.082

Dong, B., Li, H., Sun, J., Mari, G. M., Yu, X., Ke, Y., et al. (2019b). Development of a fluorescence immunoassay for highly sensitive detection of amantadine using the nanoassembly of carbon dots and $\mathrm{MnO} 2$ nanosheets as the signal probe. Sensor Actuat. B Chem. 286, 214-221. doi: 10.1016/j.snb.2019.01.100

Fernandes, D., Heslop, K. A., Kelarakis, A., Krysmann, M. J., and Estevez, L. (2020). In situ generation of carbon dots within a polymer matrix. Polymer 188:122159. doi: $10.1016 /$ j.polymer.2020.122159

Fiorani, A., Merino, J. P., Zanut, A., Criado, A., Valenti, G., Prato, M., et al. (2019). Advanced carbon nanomaterials for electrochemiluminescent biosensor applications. Curr. Opin. Electrochem. 16, 66-74. doi: 10.1016/j.coelec.2019.04.018

Fu, Y., Gao, G., and Zhi, J. (2019). Electrochemical synthesis of multicolor fluorescent $\mathrm{N}$-doped graphene quantum dots as a ferric ion sensor and their application in bioimaging. J. Mater. Chem. B 7, 1494-1502. doi: 10.1039/C8TB03103G

Gao, N., Huang, L., Li, T., Song, J., Hu, H., Liu, Y., et al. (2020). Application of carbon dots in dye-sensitized solar cells: A review. J. App. Polym. Sci. 137:48443. doi: 10.1002/app.48443

Gao, X., Du, C., Zhuang, Z., and Chen, W. (2016). Carbon quantum dotbased nanoprobes for metal ion detection. J. Mater. Chem. C 4, 6927-6945. doi: 10.1039/C6TC02055K

He, M., Guo, X., Huang, J., Shen, H., Zeng, Q., and Wang, L. (2018). Mass production of tunable multicolor graphene quantum dots from an energy resource of coke by a one-step electrochemical exfoliation. Carbon 140, 508-520. doi: 10.1016/j.carbon.2018.08.067

He, P., Shi, Y., Meng, T., Yuan, T., Li, Y., Li, X., et al. (2020). Recent advances in white light-emitting diodes of carbon quantum dots. Nanoscale 12, 4826-4832. doi: $10.1039 /$ C9NR10958G
He, S., Turnbull, M. J., Nie, Y., Sun, X., and Ding, Z. (2018). Band structures of blue luminescent nitrogen-doped graphene quantum dots by synchrotron-based XPS. Surf. Sci. 676, 51-55. doi: 10.1016/j.susc.2018.01.013

Huang, J., Liu, J., and Wang, J. (2020). Optical properties of biomassderived nanomaterials for sensing, catalytic, biomedical and environmental applications. TrAC Trends Anal. Chem. 124, 115800. doi: $10.1016 /$ j.trac.2019.115800

Iravani, S., and Varma, R. S. (2020). Green synthesis, biomedical and biotechnological applications of carbon and graphene quantum dots. a review. Environ. Chem. Lett. 18, 703-727. doi: 10.1007/s10311-020-00984-0

Jiang, K., Sun, S., Zhang, L., Lu, Y., Wu, A., Cai, C., et al. (2015a). Red, green, and blue luminescence by carbon dots: full-color emission tuning and multicolor cellular imaging. Angew. Chem. Int. Ed. 54, 5360-5363. doi: $10.1002 /$ anie. 201501193

Jiang, K., Sun, S., Zhang, L., Wang, Y., Cai, C., and Lin, H. (2015b). Bright-yellow-emissive N-doped carbon dots: preparation, cellular imaging, and bifunctional sensing. ACS Appl. Mater. Interfaces 7, 23231-23238. doi: 10.1021 /acsami.5b07255

Kour, R., Arya, S., Young, S.-J., Gupta, V., Bandhoria, P., and Khosla, A. (2020). Review-recent advances in carbon nanomaterials as electrochemical biosensors. J. Electrochem. Soc. 167:037555. doi: 10.1149/1945-7111/ab6bc4

Li, M., Chen, T., Gooding, J. J., and Liu, J. (2019). Review of carbon and graphene quantum dots for sensing. ACS Sens. 4, 1732-1748. doi: 10.1021/acssensors.9b00514

Lim, S. Y., Shen, W., and Gao, Z. (2015). Carbon quantum dots and their applications. Chem. Soc. Rev. 44, 362-381. doi: 10.1039/C4CS00269E

Liu, G., Zhao, J., Wang, S., Lu, S., Sun, J., and Yang, X. (2020). Enzyme-induced in situ generation of polymer carbon dots for fluorescence immunoassay. Sensors Actuat. B Chem. 306:127583. doi: 10.1016/j.snb.2019.127583

Liu, W., Li, C., Ren, Y., Sun, X., Pan, W., Li, Y., et al. (2016). Carbon dots: surface engineering and applications. J. Mater. Chem. B 4, 5772-5788. doi: 10.1039/С6TB00976J

Liu, Y., Duan, W., Song, W., Liu, J., Ren, C., Wu, J., et al. (2017). Red emission B, N, $\mathrm{S}$-co-doped carbon dots for colorimetric and fluorescent dual mode detection of Fe3+ ions in complex biological fluids and living cells. ACS Appl. Mater. Interfaces 9, 12663-12672. doi: 10.1021/acsami.6b15746

Liu, Y., Gou, H., Huang, X., Zhang, G., Xi, K., and Jia, X. (2020). Rational synthesis of highly efficient ultra-narrow red-emitting carbon quantum dots for NIR-II two-photon bioimaging. Nanoscale 12, 1589-1601. doi: 10.1039/C9NR09524A

Liu, Z., Zou, H., Wang, N., Yang, T., Peng, Z., Wang, J., et al. (2018). Photoluminescence of carbon quantum dots: coarsely adjusted by quantum confinement effects and finely by surface trap states. Sci. China Chem. 61, 490-496. doi: 10.1007/s11426-017-9172-0

Ma, X., Li, S., Hessel, V., Lin, L., Meskers, S., and Gallucci, F. (2019). Synthesis of luminescent carbon quantum dots by microplasma process. Chem. Eng. Process. 140, 29-35. doi: 10.1016/j.cep.2019.04.017

Molaei, M. J. (2020). Principles, mechanisms, and application of carbon quantum dots in sensors: a review. Anal. Methods 12, 1266-1287. doi: 10.1039/C9AY02696G

Mondal, T. K., and Saha, S. K. (2019). Facile approach to synthesize nitrogenand oxygen-rich carbon quantum dots for $\mathrm{pH}$ sensor, fluorescent indicator, and invisible ink applications. ACS Sustainable Chem. Eng. 7, 19669-19678. doi: 10.1021/acssuschemeng.9b04817

Nabel, G. J., and Shiver, J. W. (2019). All for one and one for all to fight flu. Nature 565, 29-31. doi: 10.1038/d41586-018-07654-w

Namdari, P., Negahdari, B., and Eatemadi, A. (2017). Synthesis, properties and biomedical applications of carbon-based quantum dots: an updated review. Biomed. Pharmacother. 87, 209-222. doi: 10.1016/j.biopha.2016. 12.108

Panwar, N., Soehartono, A. M., Chan, K. K., Zeng, S., Xu, G., Qu, J., et al. (2019). Nanocarbons for biology and medicine: sensing, imaging, and drug delivery. Chem. Rev. 119, 9559-9656. doi: 10.1021/acs.chemrev.9b00099

Pinto, D., Park, Y. J., Beltramello, M., Walls, A. C., Tortorici, M. A., Bianchi, S., et al. (2020). Cross-neutralization of SARS-CoV-2 by a human monoclonal SARS-CoV antibody. Nature 583, 290-295. doi: 10.1038/s41586-020-2349-y

Shen, P., and Xia, Y. (2014). Synthesis-modification integration: one-step fabrication of boronic acid functionalized carbon dots for fluorescent blood sugar sensing. Anal. Chem. 86, 5323-5329. doi: 10.1021/ac5001338 
Shi, R., Shan, C., Duan, X., Chen, Z., Liu, P., Song, J., et al. (2020). A human neutralizing antibody targets the receptor binding site of SARS-CoV-2. Nature 584, 120-124. doi: 10.1038/s41586-020-2381-y

Singh, V., Rawat, K. S., Mishra, S., Baghel, T., Fatima, S., John, A. A., et al. (2018). Biocompatible fluorescent carbon quantum dots prepared from beetroot extract for in vivo live imaging in C. elegans and $B A L B / c$ mice. J. Mater. Chem. B 6, 3366-3371. doi: 10.1039/С8TB00503F

Smith, B. R., and Gambhir, S. S. (2017). Nanomaterials for in vivo imaging. Chem. Rev. 117, 901-986. doi: 10.1021/acs.chemrev.6b00073

Sun, Y.-P., Zhou, B., Lin, Y., Wang, W., Fernando, K. A. S., Pathak, P., et al. (2006). Quantum-sized carbon dots for bright and colorful photoluminescence. J. Am. Chem. Soc. 128, 7756-7757. doi: 10.1021/ja062677d

Tajik, S., Dourandish, Z., Zhang, K., Beitollahi, H., Le, Q. V., Jang, H. W., et al. (2020). Carbon and graphene quantum dots: a review on syntheses, characterization, biological and sensing applications for neurotransmitter determination. RSC Adv. 10, 15406-15429. doi: 10.1039/D0RA00799D

Venkateswara Raju, C., Kalaiyarasan, G., Paramasivam, S., Joseph, J., and Senthil Kumar, S. (2020). Phosphorous doped carbon quantum dots as an efficient solid state electrochemiluminescence platform for highly sensitive turn-on detection of $\mathrm{Cu} 2+$ ions. Electrochim. Acta 331:135391. doi: 10.1016/j.electacta.2019.135391

Wang, X., Feng, Y., Dong, P., and Huang, J. (2019). A mini review on carbon quantum dots: preparation, properties, and electrocatalytic application. Front. Chem. 7:671. doi: 10.3389/fchem.2019.00671

Wang, Y., Zhu, Y., Yu, S., and Jiang, C. (2017). Fluorescent carbon dots: rational synthesis, tunable optical properties and analytical applications. RSC Adv. 7, 40973-40989. doi: 10.1039/C7RA07573A

Wang, Z., and Dai, Z. (2015). Carbon nanomaterial-based electrochemical biosensors: an overview. Nanoscale 7, 6420-6431. doi: 10.1039/C5NR00585J

Wang, Z., Wen, K., Zhang, X., Li, X., Wang, Z., Shen, J., et al. (2018). New hapten synthesis, antibody production, and indirect competitive enzymelinked immnunosorbent assay for amantadine in chicken muscle. Food Anal. Methods 11, 302-308. doi: 10.1007/s12161-017-1000-5

Wei, Y., Chen, L., Wang, J., Liu, X., Yang, Y., and Yu, S. (2020). Rapid synthesis of B-N co-doped yellow emissive carbon quantum dots for cellular imaging. Opt. Mater. 100:109647. doi: 10.1016/j.optmat.2019.109647

Wu, P., Li, W., Wu, Q., Liu, Y., and Liu, S. (2017). Hydrothermal synthesis of nitrogen-doped carbon quantum dots from microcrystalline cellulose for the detection of Fe3+ ions in an acidic environment. RSC Adv. 7, 44144-44153. doi: 10.1039/C7RA08400E

Xiao, P., Ke, Y., Lu, J., Huang, Z., Zhu, X., Wei, B., et al. (2018). Photoluminescence immunoassay based on grapefruit peel-extracted carbon quantum dots encapsulated into silica nanospheres for p53 protein. Biochem. Eng. J. 139, 109-116. doi: 10.1016/j.bej.2018.08.012

Yang, G., Wan, X., Su, Y., Zeng, X., and Tang, J. (2016). Acidophilic S-doped carbon quantum dots derived from cellulose fibers and their fluorescence sensing performance for metal ions in an extremely strong acid environment. J. Mater. Chem. A 4, 12841-12849. doi: 10.1039/C6TA05943K

Yang, S., Sun, J., Li, X., Zhou, W., Wang, Z., He, P., et al. (2014). Largescale fabrication of heavy doped carbon quantum dots with tunablephotoluminescence and sensitive fluorescence detection. J. Mater. Chem. A 2, 8660-8667. doi: 10.1039/c4ta00860j
Yao, D., Liang, A., and Jiang, Z. (2019). A fluorometric clenbuterol immunoassay using sulfur and nitrogen doped carbon quantum dots. Microchimica Acta 186:323. doi: 10.1007/s00604-019-3431-8

Yuan, F., Yuan, T., Sui, L., Wang, Z., Xi, Z., Li, Y., et al. (2018). Engineering triangular carbon quantum dots with unprecedented narrow bandwidth emission for multicolored LEDs. Nat. Commun. 9:2249. doi: 10.1038/s41467-018-04635-5

Zhang, R., Adsetts, J. R., Nie, Y., Sun, X., and Ding, Z. (2018). Electrochemiluminescence of nitrogen- and sulfur-doped graphene quantum dots. Carbon 129, 45-53. doi: 10.1016/j.carbon.2017.11.091

Zhao, S., Lan, M., Zhu, X., Xue, H., Ng, T.-W., Meng, X., et al. (2015). Green synthesis of bifunctional fluorescent carbon dots from garlic for cellular imaging and free radical scavenging. ACS Appl. Mater. Interfaces 7, 17054-17060. doi: 10.1021/acsami.5b03228

Zhong, X., Li, X., Zhuo, Y., Chai, Y., and Yuan, R. (2020). Synthesizing anode electrochemiluminescent self-catalyzed carbon dots-based nanocomposites and its application in sensitive ECL biosensor for microRNA detection. Sensor Actuat. B Chem 305, 127490. doi: 10.1016/j.snb.2019. 127490

Zhou, B., Guo, Z., Lin, Z., Zhang, L., Jiang, B.-P., and Shen, X.-C. (2019). Recent insights into near-infrared light-responsive carbon dots for bioimaging and cancer phototherapy. Inorg. Chem. Front. 6, 1116-1128. doi: 10.1039/C9QI00201D

Zhou, J., Booker, C., Li, R., Zhou, X., Sham, T.-K., Sun, X., et al. (2007). An electrochemical avenue to blue luminescent nanocrystals from multiwalled carbon nanotubes (MWCNTs). J. Am. Chem. Soc. 129, 744-745. doi: $10.1021 /$ ja 0669070

Zhou, X., Zhu, D., Liao, Y., Liu, W., Liu, H., Ma, Z., et al. (2014). Synthesis, labeling and bioanalytical applications of a tris(2,2' -bipyridyl)ruthenium(II)based electrochemiluminescence probe. Nat. Protocols 9, 1146-1159. doi: 10.1038/nprot.2014.060

Zhu, A., Qu, Q., Shao, X., Kong, B., and Tian, Y. (2012). Carbon-dot-based dual-emission nanohybrid produces a ratiometric fluorescent sensor for in vivo imaging of cellular copper ions. Angew. Chem. Int. Ed. 51, 7185-7189. doi: 10.1002/anie.201109089

Zhuo, S., Guan, Y., Li, H., Fang, J., Zhang, P., Du, J., et al. (2019). Facile fabrication of fluorescent Fe-doped carbon quantum dots for dopamine sensing and bioimaging application. Analyst 144, 656-662. doi: 10.1039/C8AN01741G

Zhuo, S. J., Fang, J., Wang, J., and Zhu, C. Q. (2019). One-step hydrothermal synthesis of silver-doped carbon quantum dots for highly selective detection of uric acid. Methods Appl. Fluoresc. 8:015005. doi: 10.1088/2050-6120/ab5d8c

Conflict of Interest: The authors declare that the research was conducted in the absence of any commercial or financial relationships that could be construed as a potential conflict of interest.

Copyright (c) 2020 Hesari and Ding. This is an open-access article distributed under the terms of the Creative Commons Attribution License (CC BY). The use, distribution or reproduction in other forums is permitted, provided the original author(s) and the copyright owner(s) are credited and that the original publication in this journal is cited, in accordance with accepted academic practice. No use, distribution or reproduction is permitted which does not comply with these terms. 